\title{
Determination of the traction resistance of the combined working body for moldboard-free tillage
}

\author{
Sergey Solovyov ${ }^{1, *}$, Yuriy Astsaturov ${ }^{1}$, Vladimir Zhigulsky ${ }^{1}$, and Inna Sulak ${ }^{1}$ \\ ${ }^{1}$ Institute of the Service Sector and Entrepreneurship (branch) of the DSTU, 147, Shevchenko Street, \\ Shakhty 346500, Rostov region, Russia
}

\begin{abstract}
The article proposes a method for calculating the traction resistance of a universal combined working body, consisting of a rack with a chisel, loosening and flat-cutting paws. The paws can have both a straight profile and a curved profile in various combinations.
\end{abstract}

\section{Introduction}

Compaction of soil horizons is a mechanical barrier to the penetration of the root system of cultivated plants into the subsurface layers, worsens the conditions for their development. Compacted soil has a reduced infiltration capacity, which is the reason for the loss of a significant amount of water to runoff and evaporation [1-4]. The need to decompact the soil, improve its structure and conditions for the development of the root system of cultivated plants, aeration, and accumulation of moisture, led to the development of loosening technology that provides crumbling of the soil, both in the upper layer and in the subsoil horizon. In Russia and all over the world, work is underway to create new and improve existing machines [4-13].

\section{Materials and methods}

The calculation of the working bodies of tillage machines is also presented by works [1416].

A method of deep loosening of the soil was proposed, the essence of which is the complex effect of a system of deformers with the formation of a loosening zone expanding to the surface of the field. Loosening of the formation is carried out without mixing the layers and bringing them to the surface when the zones of loosening of the deformers are combined.

Let us carry out a theoretical analysis of the traction resistance of a combined working body equipped with a chisel, a stand, upper and lower flat-cutting shares, with curvilinear or flat plowshares.

The traction resistance is determined by the formula:

\footnotetext{
*Corresponding author: nemnul3@mail.ru
} 


$$
P_{1 \mathrm{~s}}=2 P_{1}+2 P_{2}+P_{3}+P_{4}+P_{5},
$$

Where $P_{1}-$ traction resistance of the share of the upper share;

$P_{2}$ - traction resistance of the share of the lower share;

$P_{3}$ - traction resistance of the chisel rack;

$P_{4}, P_{5}$ - the force required for lateral separation of the soil by the plowshares of the upper and lower paws, respectively.

The traction resistances of the plowshares of each paw are determined by the formulas:

$$
\begin{aligned}
& P_{1}=P_{1}^{\prime} \sin \gamma_{1} ; \\
& P_{2}=P_{2}^{\prime} \sin \gamma,
\end{aligned}
$$

Where $P_{1}, P_{2}$ - maximum values of the traction resistance of the ploughshare installed at an angle $\gamma_{1}, \gamma$ respectively for the upper and lower legs;

$P^{{ }_{1}}, P^{{ }^{\prime}}{ }_{2}-$ maximum values of the traction resistance of the same share when it moves in the soil perpendicular to the blade.

The basis for calculating the traction resistance of the paw is the calculation of the resistance to the movement of a straight wedge in an elastic deformable medium. As a soil model for the southern regions of Russia, we take a polydisperse system with rigid bonds of a solid component and consisting of solid and gaseous phases. G.N. Dyachenko in his work identified the minimal $P_{\text {min }}^{\prime}$ share resistance:

$$
\begin{gathered}
P_{\min }^{\prime}=\left(Q \cos \alpha+N_{V} \cos \frac{\alpha}{2}-N_{y} \cos \beta^{\prime}\right) \times \frac{\sin (\alpha+\varphi)}{\cos \varphi}+ \\
+\operatorname{tg} \varphi\left(Q+N_{V} \cos \frac{\alpha}{2}-N_{y} \cos \beta^{\prime}\right)-0,5 Q \sin 2 \alpha-N_{V} \cos \alpha \sin \frac{\alpha}{2}-N_{y} \sin \beta^{\prime}
\end{gathered}
$$

Where $Q$ - gravity of the soil located on the share;

$N_{v}$ - force of resistance to kickback of the painted volume of soil;

$N_{y}$ - total force of inertia of the soil layer on the curved surface of the share;

$\beta^{\prime}$ - force deflection angle $N_{y}$;

$\alpha$ - share cutting angle;

$\varphi$ - angle of friction of soil against steel.

According to experimental data carried out on dry cohesive soil between $P^{\prime}$ and $P_{\text {min }}^{\prime}$ the is dependency established:

$$
P^{\prime}=K_{m} P_{\min }^{\prime}
$$

The quantity $K_{m}$ depends on the thickness of the treated layer $a$ : when increasing $a$ from 0,08 to 0,16 value $K_{m}$ decreases from 2,0 to 1,5 .

Using formulas (2)-(5), can be expressed by the first two terms of the formula (1):

$$
\begin{gathered}
P_{1}=K_{m 1} \sin \gamma_{1}\left[\left(Q_{1} \cos \alpha_{1}+N_{v 1} \cos \frac{\alpha_{1}}{2}-N_{y 1} \cos \beta_{1}^{\prime}\right) \times \frac{\sin \left(\alpha_{1}+\varphi\right)}{\cos \varphi}+\right. \\
\left.+\operatorname{tg} \varphi\left(Q_{1}+N_{v} \cos \frac{\alpha_{1}}{2}-N_{y 1} \cos \beta_{1}^{\prime}\right)-0,5 Q_{1} \sin 2 \alpha_{1}-N_{v 1} \cos \alpha_{1} \sin \frac{\alpha_{1}}{2}-N_{y 1} \sin \beta_{1}^{\prime}\right]
\end{gathered}
$$




$$
\begin{gathered}
P_{2}=K_{m 2} \sin \gamma\left[\left(Q_{2} \cos \alpha_{2}+N_{v 2} \cos \frac{\alpha_{2}}{2}-N_{y 2} \cos \beta_{2}^{\prime}\right) \times \frac{\sin \left(\alpha_{2}+\varphi\right)}{\cos \varphi}+\right. \\
\left.+\operatorname{tg} \varphi\left(Q_{2}+N_{v 2} \cos \frac{\alpha_{2}}{2}-N_{y 2} \cos \beta_{2}^{\prime}\right)-0,5 Q_{2} \sin 2 \alpha_{2}-N_{v 2} \cos \alpha_{2} \sin \frac{\alpha_{2}}{2}-N_{y 2} \sin \beta_{2}^{\prime}\right] .
\end{gathered}
$$

For the considered working body with simultaneous movement in the soil of the upper and lower wedge, values $Q$ and $N_{v}$ require special theoretical research.

Consider by the two wedges $A B C$ and $D E F$ angled as $\alpha_{1}$ and $\alpha_{2}$ to the horizon and located relative to each other along the line $A M$ (rear edge of the rack), inclined to the horizon at an angle $\Theta$. The height of the rear wall of the edge of each wedge above the bottom of the furrow is respectively $h_{1}$ and $h_{2}$, the depth of travel of the cutting edges relative to the surface of the medium, respectively: $a_{l}$ and $a$ (fig. 1). For the convenience of the calculation, we assume that the density of the medium on the share is constant and equal to $\rho$.

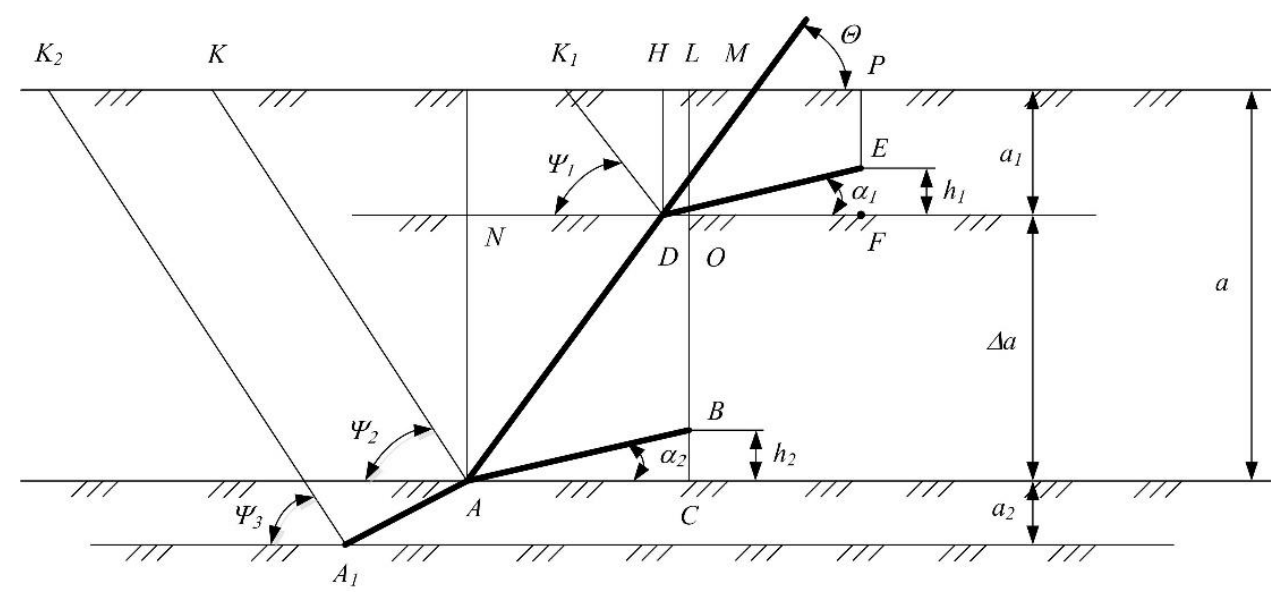

Fig. 1. Scheme for determining the magnitude of the force of the weight of the medium.

The magnitude of the force of the weight of the medium $Q$, located on the share, was determined by the formula:

$$
Q=\rho g l S,
$$

where $S$ - area of the environment above the share in the plane of the drawing, $\mathrm{m}^{2}$;

$l$ - share length, $\mathrm{m}$.

Using Figure 1, we assume that:

for top wedge

$$
S_{1}=H D \times D F
$$

for bottom wedge

$$
S_{2}=A N \times A C+Q N \times N D .
$$

Considering that $H D=a_{1}, D F=b_{1} \cos \alpha_{1}, A N=\Delta a, A C=b \cos \alpha_{2}, Q N=a_{1}, N D=\Delta a \cdot \operatorname{ctg} \Theta$ the expression (9) and (10), can be written as:

$$
S_{l}=a_{1} b_{1} \cos \alpha_{1}
$$




$$
S_{2}=\Delta a\left(b \cos \alpha_{2}+a \operatorname{lctg} \Theta\right) \text {, }
$$

Where $b_{1}, b$ - paw wing width, top and bottom, respectively.

Expression (12) fair, only if $\Delta a \leq b \cdot \operatorname{tg} \Theta \cdot \cos \alpha_{2}$. If $\Delta a>b \operatorname{tg} \Theta \cdot \cos \alpha_{2}$, then the formula (12), will take the form:

$$
S_{2}=\Delta a b \cos \alpha_{2} \text {. }
$$

Using formulas (8), (11) - (13), we matter $Q$ for each share:

$$
\begin{gathered}
Q_{1}=\rho_{1} g l_{1} a_{1} b_{1} \cos \alpha_{1} ; \\
Q_{2}=\rho_{2} g l_{2} \Delta a\left(b \cos \alpha_{2}+a_{1} \operatorname{ctg} \Theta\right), n p u \Delta a \leq b \operatorname{tg} \Theta \cos \alpha_{2} ; \\
Q_{2}=\rho_{2} g l_{2} a b \cos \alpha_{2}, n p u \Delta a>b \operatorname{tg} \Theta \cos \alpha_{2} .
\end{gathered}
$$

At the moment of the shift of the deformable volume of the medium relative to the main massif along the line $A K$ the force of resistance to displacement of the displaced part of the volume begins to act $N_{v}$. Its vector is deviated from the vertical at an angle $\alpha / 2$ according to the vector of absolute speed $V_{a}$ free incompressible bulk media. At the moment of shearing, the speed of the sheared volume increases to $V_{a}, V_{a}=2 V_{p} \sin (\alpha / 2)$. According to this force $N_{v}$ define from the expression:

$$
m V_{a}-m V_{0}=N_{v} \cdot \Delta t,
$$

where $V_{0}$ - initial velocity of a deformable medium equal to zero;

$m$ - mass of a deformed medium, separated from the main body and lying on a wedge.

The time $\Delta t$ defined as the ratio of the lifting height $h(h=b \sin \alpha)$ to the absolute velocity of the formation moving at an angle $\alpha / 2$ in relation to the vertical:

$$
\Delta t=\frac{h}{V_{a} \cos \frac{\alpha}{2}}=\frac{h}{V_{p} \sin \alpha}=\frac{b}{V_{p}} .
$$

After substitution in expression (17) values of the quantities included in it, the formula for determining the force $N_{v}$, takes the form:

for the upper paw:

$$
N_{V 1}=2 \rho_{1} l_{1} a_{1} V_{P 1}^{2} \sin \frac{\alpha_{1}}{2} \cos \alpha_{1},
$$

for the lower paw:

at $\Delta a \leq b \operatorname{tg} \Theta \cos \alpha_{2}$

$$
N_{V 2}=\frac{2 V_{P 2}^{2} \sin \frac{\alpha_{2}}{2} \rho_{1} l_{2} \Delta a\left(b \cos \alpha_{2}+a_{1} \operatorname{ctg} \Theta\right)}{b}
$$

at $\Delta a>b \operatorname{tg} \Theta \cos \alpha_{2}$

$$
N_{V 2}=2 \rho_{2} l_{2} a V_{P 2}^{2} \sin \frac{\alpha_{2}}{2} \cos \alpha_{2} .
$$

Formation inertia for each foot: at $\Delta a \leq b \operatorname{tg} \Theta \cos \alpha_{2}$ 


$$
N_{y 2}=\frac{0,5 \Delta a l_{2} \rho_{2}\left(b \cos \alpha_{2}+a_{1} c \operatorname{tg} \Theta\right) V_{P 2}^{2} \sin ^{2} \gamma}{R} ;
$$

at $\Delta a>b \operatorname{tg} \Theta \cos \alpha_{2}$

$$
\begin{aligned}
N_{y 2} & =\frac{0,5 a b l_{2} \rho_{2} V_{P 2}^{2} \sin ^{2} \gamma}{R} . \\
N_{y 1} & =\frac{0,5 a_{1} b_{1} l_{1} \rho_{1} V_{P 1}^{2} \sin \gamma_{1}}{R},
\end{aligned}
$$

Where $R=r_{0} e^{a \varphi_{n}} \sqrt{1+a^{2}}, \beta^{\prime}=\frac{\pi}{4}-\varphi_{n}$.

For a curved wing profile with $\alpha_{\max }=45^{0} ; \alpha_{\min }=7 \ldots 12^{0} ; b=80 \mathrm{~mm} ; h=38 \mathrm{~mm}$, the following values are obtained: $\varphi_{n}=9^{0} 31^{\prime} ; r_{0}=1,41 \cdot 10^{-2} \mathrm{~m} ; a=1$.

Thus, the traction resistance of the share $P_{2}, P_{1}$ for the lower and upper paws will be determined by the formula (6) and (7) taking into account expressions (14)-(16) and (19)(24).

In order to determine the traction resistance of the strut with the chisel, let us divide this element of the working body into two parts: the chisel and the strut. We assume that the chisel works below the critical depth of cut, and the upper part of the tine, which is above the chisel, works in the already slightly loosened soil with the lower and upper paws and therefore only moves the soil. Then the thrust resistance of the chisel rack includes two components

$$
P_{3}=P_{n}+P_{c},
$$

where $P_{n}$ - force required to press soil into the walls of the gap below the critical depth of cut;

$P_{c}$ - force required to shear the sturdy soil.

$$
P_{n}=0,5 q a_{2} b_{2}^{2},
$$

where $a_{2}-$ bit depth, $a_{2}=l_{3} \sin \alpha_{3}$;

$b_{2}$ - bit width, m;

$q$ - soil volumetric crushing coefficient, $\mathrm{N} / \mathrm{m}^{3}$

To consider the shear force of the soil $P_{c}$ we will divide the rack into two parts, then:

$$
P_{c}=P_{c 1}+P_{c 2},
$$

where $P_{c l}$ - the shear force of the soil by the strut positioned above the upper paw, at a depth $a_{1}$;

$P_{c 2}$ - force of shearing of the soil by the stable, located above the lower paw, at a depth $\Delta a$.

In accordance with the expression for the force for a stable soil shear, we will have:

$$
\begin{aligned}
P_{c 1} & =\frac{C_{01} h_{1} \cos \varphi\left(b_{3} c \operatorname{tg} \omega_{1}+h_{1}\right)}{\sin \psi_{1} c t g \omega_{1} \cos \left(\psi_{1}+\varphi\right)\left(1-\operatorname{tg} \varphi_{1}\right) \operatorname{tg}\left(\psi_{1}+\varphi\right)} ; \\
P_{c 2} & =\frac{C_{02} h_{2} \cos \varphi\left(b_{3} c \operatorname{tg} \omega+h_{2}\right)}{\sin \psi_{2} \operatorname{ctg} \omega \cos \left(\psi_{2}+\varphi\right)\left(1-\operatorname{tg} \varphi_{1}\right) \operatorname{tg}\left(\psi_{2}+\varphi\right)},
\end{aligned}
$$

Where $C_{0}$ - soil connectivity, $\mathrm{N} / \mathrm{m}^{2}$;

$\omega-$ lateral soil separation angle;

$\varphi_{1}-$ soil friction angle; 
$b_{3}-$ rack thickness.

The forces required for lateral separation of the soil by the share of the upper and lower paws $P_{4}$ and $P_{5}$ will be determined in accordance with the methodology described in [5]:

$$
\begin{gathered}
P_{4}=\sigma_{\mathrm{p}}\left[R_{1}\left(R_{1}-\frac{a_{1}}{\cos \varphi}\right)\left(R_{1}-\frac{a_{1}}{\cos \omega_{1}}\right)\left(R_{1}-a_{1} \sqrt{\operatorname{ctg}^{2} \varphi+\operatorname{ctg}^{2} \omega_{1}}\right)\right]^{1 / 2} ; \\
P_{5}=\sigma_{\mathrm{p}}\left[R_{2}\left(R_{2}-\frac{a}{\cos \varphi}\right)\left(R_{2}-\frac{a}{\cos \omega}\right)\left(R_{2}-a \sqrt{\operatorname{ctg}^{2} \varphi+\operatorname{ctg}^{2} \omega}\right)\right]^{1 / 2},
\end{gathered}
$$

where $\sigma_{p}$ - permissible shear stress, $\mathrm{N} / \mathrm{m}^{2}$

$R_{i}$-coefficient taking into account the depth of cultivation and soil properties:

$$
\begin{aligned}
& R_{1}=0,5 a_{1}\left(\frac{1}{\cos \psi_{1}}+\frac{1}{\cos \omega_{1}}+\sqrt{\operatorname{ctg}^{2} \varphi+\operatorname{ctg}^{2} \omega_{1}}\right) \\
& R_{2}=0,5 a\left(\frac{1}{\cos \psi_{2}}+\frac{1}{\cos \omega}+\sqrt{\operatorname{ctg}^{2} \varphi+\operatorname{ctg}^{2} \omega}\right) .
\end{aligned}
$$

\section{Conclusion}

As a result of the theoretical analysis, it is possible to calculate the traction resistance of the combined working body for moldboard-free tillage. It is first necessary to carry out experiments to identify the values of the coefficient $K_{m}$ depending on the depth of the processed layer $a_{l}$ for the considered working body.

\section{References}

1. A. Mamkagh, Asian Journal of Advances in Agricultural Research 9(1), 1-7 (2019) https://doi.org/10.9734/AJAAR/2019/46706

2. A. Chappell, N. Webb, Aeolian Research 23, 63-78 (2016) https://doi.org/10.1016/j.aeolia.2016.09.006

3. Z. Sándor, M. Tállai, I. Kincses et al., DRC Sustainable Future 1(1), 14-20 https://doi.org/10.37281/DRCSF/1.1.3

4. G. Parkhomenko, I. Bozhko, S. Kambulov et al., E3S Web of Conferences 175, 09006 (2020) https://doi.org/10.1051/e3sconf/202017509006

5. G. Parkhomenko, S. Kambulov, V. Pakhomov et al., E3S Web Conf. 210, 08007 (2020) DOI: 10.1051/e3sconf/202021008007

6. B.A. Volik, A.I. Babble, A.V. Konoviy, Environmental Engineering 2(10), 45-48 (2018) http://enm.khntusg.com.ua/index.php/enm/article/view/87

7. Y. Lachuga, B. Akhalaya, Y. Shogenov et al., E3S Web Conf. 210, 08016 (2020) DOI: $10.1051 / \mathrm{e} 3$ sconf $/ 202021008016$

8. A. Drovnikov, B. Kalmykov, E3S Web Conf. 210, 08003 (2020) DOI: 10.1051/e3sconf/202021008003

9. V.V. Ivanov, S.I. Popov, N.S. Dontsov, Ju.V. Marchenko, N.V. Ryzhkin, Ju.A. Oleynikova, XVI International scientific-technical conference «Dynamics of technical 
systems» (DTS-2020): IOP Conference Series: Materials Science and Engineering 1029, 012052 (2021) doi:10.1088/1757-899X/1029/1/012052

10. V.V. Ivanov, S.I. Popov, N.S. Dontsov, G.E. Ekinil, Ju.A. Oleynikova, Ju.N. Denisenko, XIII International Scientific and Practical Conference «State and Prospects for the Development of Agribusiness - INTERAGROMASH 2020»: E3S Web of Conferences 175, 05023 (2020) doi.org/10.1051/e3sconf/202017505023

11. S. Solovyov, E. Milutin, V. Ryzhikov, Improvement of the internal combustion engine control system, 1-4 (2017) DOI:10.1109/EWDTS.2017.8110083

12. A. Buryanov, V. Ignatenko, I. Ignatenko, I. Vyalikov, MATEC Web Conf. 226, 01029 (2018) DOI: $10.1051 /$ matecconf/201822601029

13. V.A. Ryzhikov, Y.G. Sapronov, S.L. Gorin, Y.G. Astsaturov, ARPN Journal of Engineering and Applied Sciences 10(12), 5334-5337 (2015)

14. S.I. Kambulov, I.V. Bozhko, A.V. Olshevskaya, MATEC Web of Conferences 224, 05022 (2018) https://doi.org/10.1051/matecconf/201822405022

15. I. Bozhko, G. Parkhomenko, S. Kambulov et al., E3S Web of Conferences 175, 05025 (2020) INTERAGROMASH 2020. https://doi.org/10.1051/e3sconf/202017505025

16. A. Altybayev, A. Zhanbyrbayev, B. Meskhi, et al., E3S Web of Conferences 135, 01078 (2019) https://doi.org/10.1051/e3sconf/201913501078 